\title{
An Energy-based Wireless Sensor Network Model to Improve the Performance of the Internet of Things
}

\author{
Sharaf Alhomdy \\ Associate Professor \\ Faculty of Computer and \\ Information Technology, \\ Sana'a University, Sana'a, Yemen
}

\author{
Mohammed Q. AL_shamiry \\ Faculty of Computer and \\ Information Technology, \\ Sana'a University, Sana'a, Yemen
}

\author{
Ghaleb Al-Gafary \\ Professor \\ Faculty of Computer and \\ Information Technology, \\ Sana'a University, Sana'a, Yemen
}

\begin{abstract}
Internet of Things (IoT) plays an important role by bringing together people, process, data, and things to make networked connections more relevant and valuable. The devices of the IoTs sense environmental conditions using Wireless Sensor Networks (WSNs), so WSN are the most important components of the IoT. WSN is a widespread network used in many IoT applications and any improvement in WSN reflects positively on IoT. There are many important issues related to WSN such as energy consumption, end-to-end delay, and packet loss rate. This paper aims to propose efficient control system model to improve the Quality of Service (QoS) requirements and maximize the resource utilization in WSN, through adapts the data rate that should be used by the source nodes, and determine the appropriate routing protocol to work in the network according to the network conditions. Castalia 3.2 and XEEMU-1.0 simulators were used to evaluate the control system model performance with taking into account different network scenarios. The simulation results showed the efficiency of the proposed Model in terms of reducing power consumption, end-to-end delay, and packet loss rate compared to the other schemes.
\end{abstract}

\section{Keywords}

Wireless Sensor Networks, Model,Internet of Things, Quality of Service

\section{INTRODUCTION}

Internet of Things (IoT) is a concept and a paradigm that considers pervasive presence in the environment of a variety of things/objects through wireless connections using unique addressing schemes which able to interact with each other and cooperate with other things/objects to create new applications/services to reach common goals. It is a technological revolution that represents the future of computing and communications. It aims toincrease the ubiquity of the Internet by integrating every object for interaction via embedded systems, which leads to highly distributed network of devices that communicate and sense with their internal states or the external environment such as human beings andother devices. Indeed, sensors are the bridge that connects the physical world to the digital-world through the transmission of sensed physical values across the network to the end-user [1][2].

In IoT environment, the Wireless Sensor Networks (WSNs) is themost important components where sensors serve as hands and feet for the IoTs within sensors network. WSNs are consists of sensors which are distributed in an ad hoc manner, these sensors work with each other to sense some physical phenomenon and then the information gathered is processed to get relevant results. So, there are some challenges in WSN: sensors have a limited power source, data transmission delay and data transmitted loss [2][3]. Some research studies handled some of these challenges in order to increase the lifetime of the network, and to guarantee the best possible QoS through some common basic parameters, such as the energy efficiency, end-to-end delay, and packet loss rate. However, they still have some limitation in terms of the performance the network concerning with these parameters [4][5].

In this paper, a control system model was proposed and applied for providing QoS, it guarantees efficient resource utilization in WSNs which in turn enhances IoTs performance.The control system model was used to keep track of the network conditions and resources using information derived from different layers, adapted the data transmission accordingly, and determined the appropriate routing protocol in order to satisfy the QoS requirements and maximize the network lifetime.

The rest of the paper is described as follows: Section 2 conducts a related studyabout IoT, WSN, cross-layering in WSN, and the fuzzy logic techniques, and gives some related work. Section 3 describes the proposed control system model to improve the QoS in WSNs. performance evaluation and discusses the simulation results is provided in section 4 . Finally, section 5 draws the conclusion and recommendations.

\section{RELATED STUDY}

The WSN is most important components in IoT environment, the WSN consists of a group of small sensors that have a limited power source. Energy consumption is one of the most important issues related to the lifetime of WSN. There are sensors that collect data, and other sensors that receive data from sensors and send them to the Base Station (BS), that are considered Cluster Header $(\mathrm{CH})$. The WSN is associated with three important factors that affect negatively its performance which are end-to-end delay,energy consumption, and Packets Delivery Ratio. The delay in the transmission of data may occur between sensors themselves or between sensors and the BS. There are many other threats and factors affecting the successful access of data to BS and data loss which are also important problem in WSN. Many researchers have focused on improving one or more of the QoS parameters, such as energy consumption, end-to-end delay and Packets Delivery Ratio. This section provides review of different researches related to this paper as follows.

In 2018, Youngbok Cho an et al. [4] proposed hierarchical network architecture to solve the energy consumption problem, and implement suitable routing mechanism to handle low power devices (devices battery operated). The simulation results showed that the proposed scheme is more energy efficient and flexible than traditional wireless sensor network schemes and consequently, the new scheme can be implemented for efficient communication in the Internet of Things. The proposed approaches didn't focus on end-to-end delay and packets delivery ratio. 
EiP-LEACH is protocol proposed by Anupkumar M Bongale et al. [6] aims at enhance energy consumption in WSN, in which using probability based threshold value that is influenced by residual energy of candidate Cluster Header node and average initial energy of entire network for Cluster Header selection, their proposed reduces energy consumption when the distance is small between the Cluster Header and the Base Station, in their protocol only energy is considered the primary parameter in deciding Cluster Header node. But they did not care about the distance to Base Station.

Hongyan xin and Xuxun liu.[7] proposed an accurate-distances based transmission scheme, to solve the many-to-one transmission problem of strip-based Wireless Sensor Network and avoid energy holes of such a Wireless Sensor Network, which is divided into multiple layers. This scheme didn't care about transmission design assisted by super nodes, and they didn't care in delay in WSN.

In [8], Hui Chen et al. proposed an improved inter-cluster multihop routing protocol based on quality of service (CMPBQ), that base on traditional LEACH protocol, this protocol aims to ensure reliable data transmission in WSNs. The simulation results showed that the proposed protocol improve the data transmission reliability. In this approach, the authors didn't focus on delay and Energy consumption in WSN.

Hasna Dhehibi et al.[9] proposed new design of energy efficient LEACH protocol that based on MOD LEACH. They tried to manage intelligently the remain power of the node in order to increase lifetime of the network and packets delivery ratio. Their results demonstrated that their proposed protocol well improve lifetime of the network and packets delivery ratio to the base station. This model did not focus on end-to-end delay.

Sabin Bhandari et al.[10] proposed a new cloud-assisted clustering framework, where each sensor node in their proposed framework elects itself as a $\mathrm{CH}$ on the basis of the closeness factor, and residual energy. They analyzed the impact of heterogeneity of Internet of Things networks in terms of bondness among IoT nodes and energy. The numerical results show that the proposed framework reduces End to End delay, and provides higher throughput and longer network stability as compared to the conventional one. The proposed framework is not suitable for Internet of Vehicle (IoV) network, the proposed framework doesn't prioritization for wireless IoT.

In [11] Mengkun et al. proposed a new dynamic network routing protocol for WSN, through improved the GPSR protocol based on stratification of traffic density. The main idea of improved the GPSR protocol is using a stratification strategy, depending on the density of nodes nearby, Where the nodes can adjust strategy of maintaining neighbors list. Nodes maintain a two- hops-neighbor list, when the density of nodes nearby is lower than critical value. Nodes maintain a one-hop neighbor list, when the density of nodes nearby is larger than critical value. Simulation experimental results showed the performance of improved algorithm has an improvement on average transmission delay compared with the classical GPSR algorithm, and also the transmission overhead was smaller than the 2- hop GPSR protocol. The authors did not focus on Energy consumption in WSN.

\section{PROPOSED CONTROL SYSTEM MODEL}

The control system model improves the QoS in WSNs which is reflected positively on IoTs. The key idea behind this model is to send data with different data rates that commensurate with the current conditions of the network, and to use the appropriate routing protocol according to these conditions.

\subsection{Model Overview}

The proposed model represents an adaptive control system, which controls the amount of data to be sent over the network and determines the appropriate routing protocol to work in the network. The system monitors the QoS parameters continuously then gives the most affected parameter the priority until being recovered from its deterioration.

Figure (3.1) shows the pseudo-code of the proposed model. As shown in the figure, the sink node collects information about the average packet loss, average network delay and average power consumption. This information is used as inputs to the control system model for estimating the suitable data rate, that used by the source nodes and determining the appropriate routing protocol. Then, the sink node sends control messages to the network nodes, to adapt the data rate and the appropriate routing protocol. After that, the sink node starts again collecting information from the relevant layers and analyses them using the controller system to send new control messages to the network nodes in order to keep or modify the current data rate and routing protocol.

The proposed control system model consists of fuzzy logic and binary controllers; the fuzzy controller is designed based on the Mamdani model. The input to the proposed control system model are the average of remaining power in the network, the average of loss rate, and the average of network delay as shown in figure (3.2).

1. Deploy $\mathrm{N}$ sensor nodes in a sensing field, where some of the sensors are sources.

2. Data Rate $\mathrm{R}=$ Original Data Rate

3. Routing Protocol $=$ GPSR

4. For each source sensor do:

5. Collect data $\mathrm{D}$.

6. Send D with Data Rate R, using the appropriate Routing Protocol.

7. Wait for control message from the base station (the sink node).

8. EndFor

9. Receive information from the source nodes at base station.

10. Determine, using control system, the appropriate Routing Protocol, and the new Data Rate R.

11. Send control message to adjust Data Rate $R$, and Routing Protocol to the source nodes and to relay nodes.

12. Go to 4.

Fig. (3.1): Pseudocode for the Proposed Model 


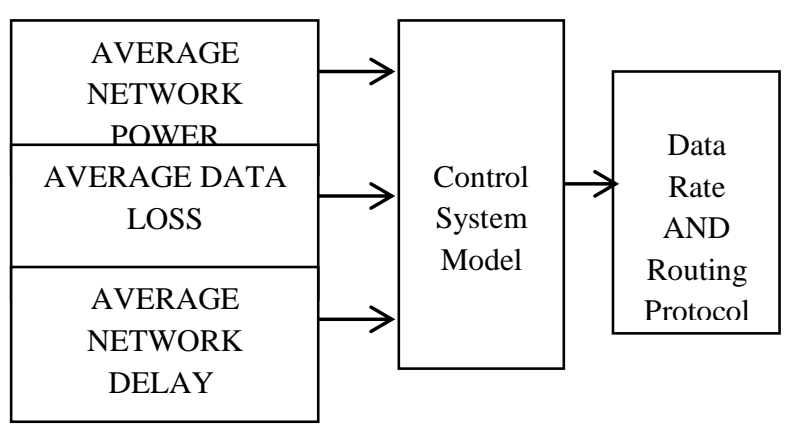

Fig.(3.2): Control System Model Architecture

Each input represents a fuzzy variable, which is represented by fuzzy sets; these inputs are used for the fuzzy logic controller, which uses them to estimate the suitable data rate, that used by the source nodes.In fuzzy controller, the AND operator was used to combine the three inputs in the IF-THEN rules; "IF $\mathrm{x}$ is A AND y is B THEN Data Rate is C"; as a result, there are 60 different rules, as shown in Table 1 , to cover all the possible conditions.

Table 1: Fuzzy Controller Rules

\begin{tabular}{||l|l|l|l|l||}
\hline \multicolumn{5}{|c|}{ Table 1: Fuzzy Controller Rules } \\
\hline 1 & NET.POWER & DELAY & LOSS & Data Rate \\
\hline 2 & HIGH & VHIGH & EHIGH & VERYLOW \\
\hline 3 & HIGH & VHIGH & VHIGH & LOW \\
\hline 4 & HIGH & VHIGH & HIGH & MEDUIM \\
\hline 5 & HIGH & VHIGH & LOW & VERYHIGH \\
\hline 6 & HIGH & HIGH & EHIGH & VERYLOW \\
\hline 7 & HIGH & HIGH & VHIGH & LOW \\
\hline 8 & HIGH & HIGH & HIGH & MEDUIM \\
\hline 9 & HIGH & HIGH & MEDUIM & HIGH \\
\hline 10 & HIGH & HIGH & LOW & VERYHIGH \\
\hline 11 & HIGH & MEDUIM & EHIGH & VERYLOW \\
\hline 12 & HIGH & MEDUIM & VHIGH & LOW \\
\hline 13 & HIGH & MEDUIM & HIGH & MEDUIM \\
\hline 14 & HIGH & MEDUIM & MEDUIM & HIGH \\
\hline 15 & HIGH & MEDUIM & LOW & VERYHIGH \\
\hline 16 & HIGH & LOW & EHIGH & VERYLOW \\
\hline 17 & HIGH & LOW & VHIGH & LOW \\
\hline 18 & HIGH & LOW & HIGH & MEDUIM \\
\hline 19 & HIGH & LOW & MEDUIM & HIGH \\
\hline 20 & HIGH & LOW & LOW & VERYHIGH \\
\hline 21 & MEDUIM & VHIGH & EHIGH & VERYLOW \\
\hline 22 & MEDUIM & VHIGH & VHIGH & VERYLOW \\
\hline 23 & MEDUIM & VHIGH & HIGH & LOW \\
\hline 24 & MEDUIM & VHIGH & MEDUIM & MEDUIM \\
\hline 25 & MEDUIM & VHIGH & LOW & HIGH \\
\hline 26 & MEDUIM & HIGH & EHIGH & VERYLOW \\
\hline 27 & MEDUIM & HIGH & VHIGH & LOW \\
\hline 28 & MEDUIM & HIGH & HIGH & LOW \\
\hline 29 & MEDUIM & HIGH & MEDUIM & HIGH \\
\hline 30 & MEDUIM & HIGH & LOW & VERYHIGH \\
\hline
\end{tabular}

Whereas, the switching operation between the suitable routing protocol is carried out using binary controller. The binary controller is dedicated for switching the routing protocol between GPSR and LEACH whenever network remaining power average getting to be less than $50 \%$ of the initial power. This policy decreases the network power consumption operating together with the fuzzy controller, which is dedicated to decrease network power consumption by adapting network data rate.

\subsection{Cross-Layer System Architecture}

Figure (3.3) shows the cross-layer system architecture. As shown, the control system model is designed according to the bottom up architecture, in which the upper layers change their traditional behaviors in order to adapt to the changes occurred in the lower layers. The layers that are involved in the system architecture are the physical layer, the network layer, and the application layer. The control system model sends frequent control messages to the application layers of the source nodes, and to network layers in all network nodes to change their behaviors according to the information obtained from the other layers. 
This system deals with the average of power consumed by all the network nodes, not for each node independently, to prevent the nodes from changing their own behaviors independently from each other which may make variations in routing protocol used in the network.

\subsubsection{The Average of Network Residual Power Input}

This input is provided by the physical layer. It indicates the amount of remaining power in the network. It uses the membership functions of the network residual power fuzzy variable for three fuzzy sets: low residual power, medium residual power, and high residual power, with total range of 100 units that represents the percentage of the average network power for all the nodes [12][13].

Routing Protocol

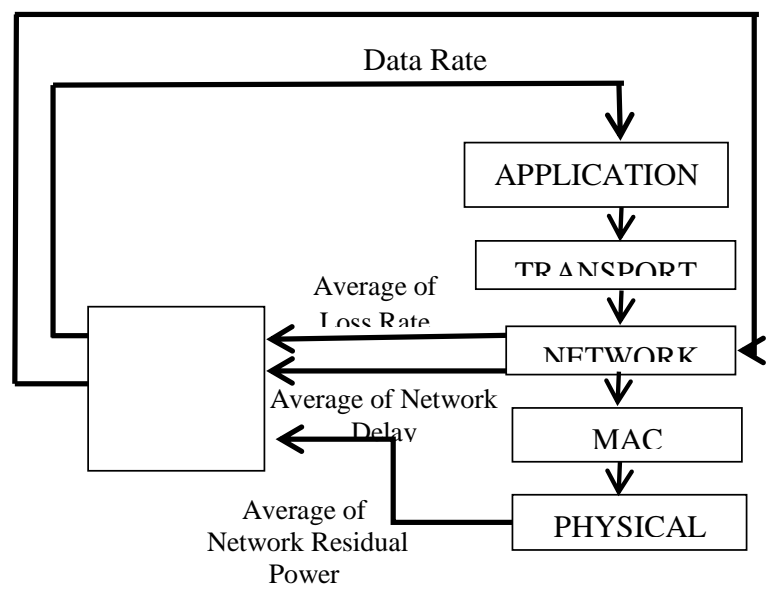

Fig.(3.3): Cross-layer System Architecture

\subsubsection{The Average of Loss Rate Input}

This input is provided by the network layer. It uses the membership functions of the average loss rate for five member functions (low, medium, high, very high, and extremely high). The ranges of the membership functions are selected according to results obtained from different scenarios with different conditions which give us an idea about the effect of the network conditions on these parameters. Also this classification was considered by other studies after studying the effect of the loss rate on the quality of the data at the receiver side

\subsubsection{The Average of Network Delay Input}

The third fuzzy input is the average network delay. It is provided by the network layer. The acceptable network delay depends on the application and the network topology as well as on the underground channel conditions. The fuzzy sets of the average delay input which consists of four member functions (low, medium, high, and very high) is used. This manner of set division is considered according to other studies were aiming to reduce the network congestion by using the delay factor as an indicator of the network congestion [12].

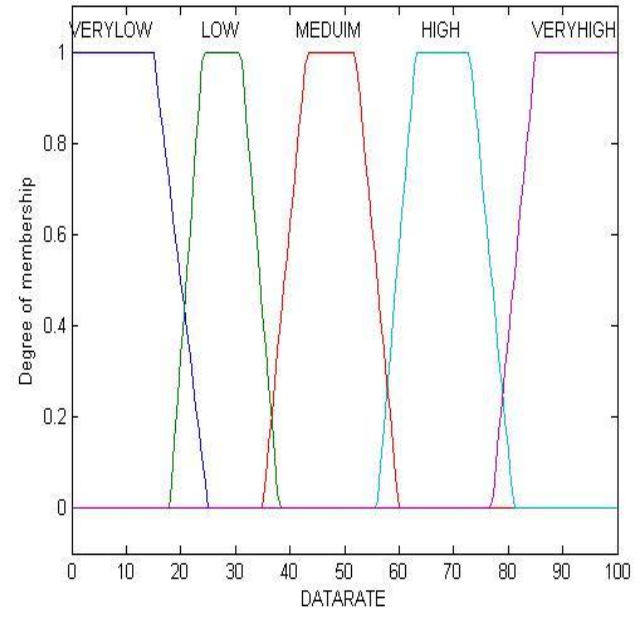

Fig. (3.4) : Membership Functions of the Output Variable (Data Rate)

\subsubsection{The Data Rate Output}

Figure (3.4) shows the fuzzy sets of the data rate as a fuzzy output. As shown, these sets are symmetrically divided to form five membership functions ranging from very low to very high, (very low, low, medium, high, and very high), with total range of 100 units. Each fuzzy set represents a set of data rates and the defuzzification value based on the Mamdani model [14] as shown in equation (1) is mapped to one of 39 different data rates ranging between $24 \%$ and $100 \%$.

$\operatorname{defuzzification}(\mathrm{C})=\frac{\sum_{\mathbf{j}=1}^{\mathbf{N}} \mathbf{z}_{\mathrm{j}} \mu_{\mathrm{C}}\left(\mathbf{z}_{\mathrm{j}}\right)}{\sum_{\mathbf{j}=1}^{\mathbf{N}} \mu_{\mathrm{C}}\left(\mathbf{z}_{\mathrm{j}}\right)}$

Equation(1): Mamdani model's mathematical equation [14]

Where $\mu$ Crisp Input, Zj Degree Of Membership, and C fuzzy set.

\section{IMPLEMENTATION AND RESULTS DISCUSSIONS}

In this section the performance of the proposed model was evaluated. First, define the performance parameters, then, the simulation environment is described. A set of scenarios that represent different excrements were conducted, Performance Evaluation and the effect of using different data rates and different routing protocols on the performance parameters was studied by comparing the proposed model with other schemes.

\subsection{Performance Parameters}

This section defines and discusses the performance parameters which were taken into account during the system design that are the packet loss rate, network delay, and power consumption.

\subsubsection{Packet Loss Rate}

Packet loss occurs when one or more packets of data travelling across a computer network fail to reach their destination. Packet loss is either caused by errors in data transmission, typically across wireless networks, or network congestion. Packet loss is measured as a percentage of packets lost with respect to packets sent. 


\subsubsection{Network Delay}

Network delay is a design and performance characteristic of a network. It specifies the latency for a bit of data to travel across the network from one communication endpoint to another. It is typically measured in multiples or fractions of a second. Delay may differ slightly, depending on the location of the specific pair of communicating endpoints.

\subsubsection{Power Consumption}

Electric energy consumption is the form of energy consumption that uses electric energy. Electric energy consumption is the actual energy demand made on existing electricity supply.

WSN needs to provide power continuously, but the sensors have low power and energy constrained due to their small size. In this simulation, there are several factors affect the energy consumption in WSNs. The first factor is the data acquisition. In this paper, considered image capturing, which consumes around $87.9 \mathrm{mj}$ as acquisition power [15]. The second factor is the communication, which consumes more energy than any other task. Finally, there is part of the energy spent in data processing in the application layer during re-sizing the images before being sent. All these factors were considered during calculating the power consumption.

\subsection{Simulation Environment}

Using a well-equipped simulator with real data (real images) to simulate a WSNs environment can give us a useful and realistic statistic. Moreover, using more than one simulator to represent the various processing operations that may take place in the network can make the simulation more realistic. Therefore, in the simulation scenarios, more than one simulator was used to cope with the variation of the operations that must be performed in the wireless sensor networks, such as image capturing, compression, and transmission, and taking into account the power required to perform these operations. Castalia 3.2 [16] and XEEMU-1.0 [16] simulators with Matlab software and other image processing utilities such as cjpeg (available with the IJG JPEG library [17]) were used to simulate all the operations starting with image capturing until displaying the received images at the base station.

\subsubsection{Network Simulator}

Castalia is a simulator for low-power embedded devices networks such as wireless sensor networks, and body area networks. Researchers and developers can use Castalia, which is based on OMNeT++ platform [17], to test their algorithms and protocols in realistic wireless network environment especially relating to wireless channel and radio models because it supports the following features:

- Full support for node mobility.

- Handling interference as the strength of the received signal.

- Define a map of path loss.

- Support the variation of transmission power level of nodes individually.

- RSSI and carrier sensing with complex and realistic modelling.

- Different delays and power consumption states.

- Support future adaptation and expansion.

\subsubsection{Power Simulator}

Energy efficiency is an important factor in wireless sensor networks, so to make the right design decisions researcher should have an idea about the power consumption of the system in early design phases. Therefore, researcher need an accurate power simulator to simulate all the operations may be performed in the system. XEEMU is a power simulator dedicated for the Intel XScale-based systems. XEEMU is also a configurable runtime simulator with very high accuracy. The average error is $3.0 \%$ for runtime and $1.6 \%$ for the estimation of CPU energy consumption. This simulator can be used together with another application to compute the delay and power consumed during the operation of this application [16].

\subsubsection{Data Processing Utility}

Real data in this scenarios was used, which is real pictures. The cjpeg utility is used to compress an image file using IJG JPEG library. JPEG is a loss compression method, which means that the output image is not necessarily to be identical to the input image. JPEG can play well with typical real-world images. It can achieve very good compression levels with no visible change, and it can achieve high compression if the application requirements tolerate a low-quality image. By adjusting the compressor's "quality" setting, cjpeg can trade off image quality against file size. It also provides all necessary facilities to dealing with images compression and resizing [17]. Cjpeg is used for compressing and resizing images before being sent by the source nodes. In addition, it is used with XEEMU simulator to provide us with accurate information about the power consumed and processing delay during compression and resizing operations.

\subsection{Network Scenarios}

To evaluate the performance parameters of the proposed model, there are three different network scenarios have been taken in the simulation scenarios and analysis as shown in the following points.

\subsubsection{Scenario (1)}

In this scenario, 5 nodes were selected ( $10 \%$ of total nodes) to be the sources and distributed them around the sink, which is located in the middle of the region of interest. The source nodes capture images continuously at different intervals to be sent by relay nodes to the sink.

\subsubsection{Scenario (2)}

This scenario aims to simulate the network with more data congestion than the previous scenario. The network consists of 10 source nodes ( $20 \%$ of total nodes). The source nodes capture and send images with different intervals. This scenario studies the effect of increasing the amount of data to be sent through the network by increasing the number of source nodes which will transmit more images.

\subsubsection{Scenario (3)}

This scenario uses to stimulate the worst conditions that the networks may face. In this case, the network congestion was increased by increasing the number of sources to 20 nodes ( $40 \%$ of total nodes).

The following table show the configuration in the simulation tool for different scenarios.

Table (2): Simulation Configurations

\begin{tabular}{||c|c||}
\hline Parameters & Values \\
\hline Network size & $200 \times 200 \mathrm{~m}^{2}$ \\
\hline Number of nodes & 50 nodes \\
\hline Packet size & 256 bytes \\
\hline Communication range & $30 \mathrm{~m}$ \\
\hline Routing protocol & GPSR, LEACH \\
\hline MAC protocol & TMAC \\
\hline Initial energy & $50 \mathrm{~J}$ \\
\hline Simulation time & $400 \mathrm{C}$ \\
\hline
\end{tabular}

\subsection{Performance Evaluation}


In this section, the simulation results of the three scenarios are presented and discussed for different parameter as follow:

\subsubsection{Packet Loss Rate}

In wireless sensor networks, sending large amount of data can turn situation from bad to worse especially if all of the sources send their data over the network at the same time. In this case, the network may suffer from high loss rate in addition to receiving distorted or unreadable data which wastes the network resources. Figure (4.1) shows the average packet loss rate for three different scenarios named according to the number and percentage of source nodes to the total number of nodes in the network $(10 \%$ " 5 source nodes", $20 \%$ "10 source nodes", and 40\% "20 source nodes"). Figure (4.2) shows the packet loss rate as a function of time for the three scenarios. The result show that when the number of nodes increase means that the flooding the network with increase which make the network more congested this can significantly affect the amount of data being lost.

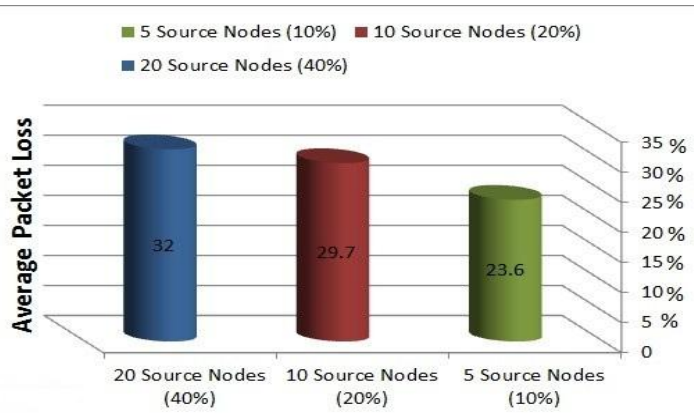

Figure (4.1):Average Packet Loss for Different Network Scenarios

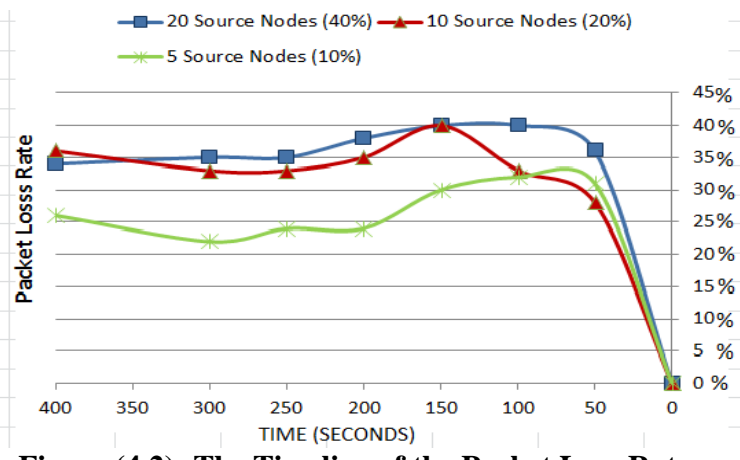

Figure (4.2): The Timeline of the Packet Loss Rate forDifferent Network Scenarios

\subsubsection{Network Delay}

Flooding the network with data may lead to congestion and bottleneck problems which affect the time that the packets need to reach their destination. In addition, considering an alternative path because of the death of some relay nodes may also affect directly the delay. Figure (4.3) shows the delay average for the three scenarios when using a different number of source nodes. Figure (5.4) shows the delay rate as a function of time for the three scenarios. As shown in figure (4.3) and figure (4.4). Increasing the number of source nodes increases the delay because of overwhelming the network with larger amount oftraffic.

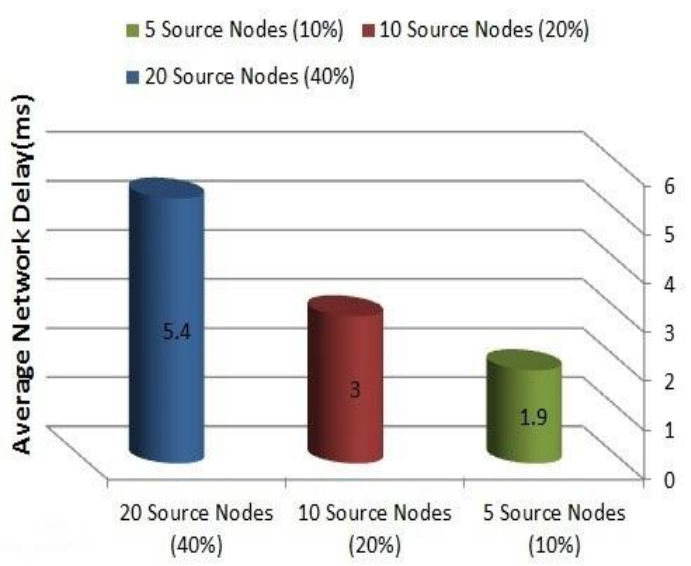

Figure(4.3) : Average Network Delay for Different Network Scenarios

\subsubsection{Power Consumption}

The higher the number of source nodes, the more data will be captured, this mean more power consumption. These data need to be processed and transmitted which requires more power. Figure (4.5) shows the average power consumed for the three scenarios when using a different number of source nodes. Figure (4.6) shows the power consumption rate as a function of time for the three scenarios. As shown in figure (4.5) and figure (4.6) increasing the number of source nodes increases the power consumption because of the increased amount of data sent over the network and that effects on the network lifetime.

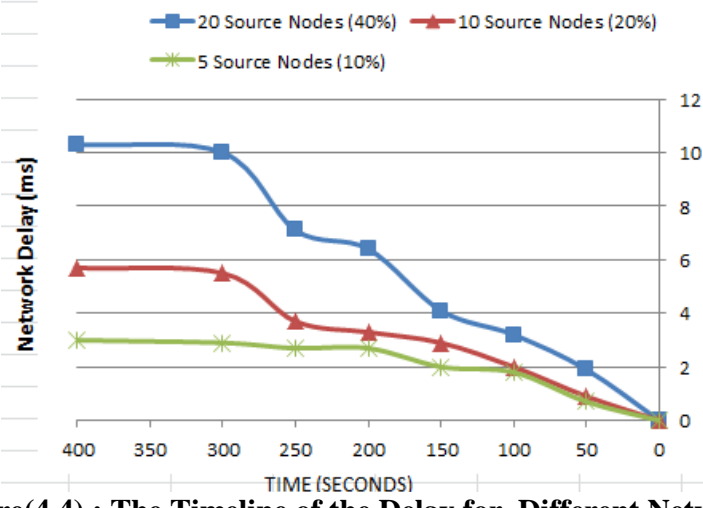

Figure(4.4) : The Timeline of the Delay for Different Network Scenarios

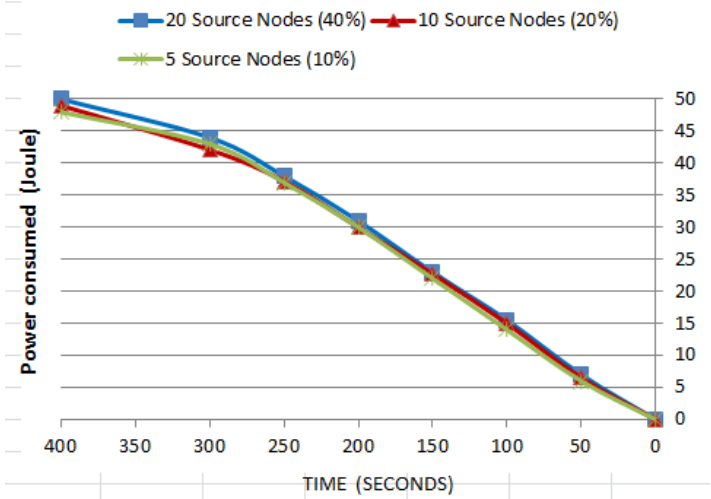

Figure(4.5) : The average power consumed for the three scenarios 


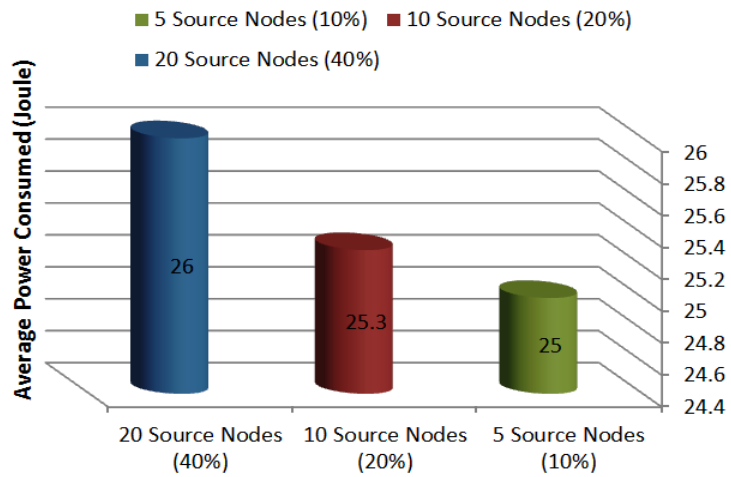

Figure (4.6) shows the power consumption rate as a function of time for the three scenarios

\subsection{Comparison with Other Methods}

To verify the proposed model, the simulation was done using simulation tools based on the following parameters; Packet Loss Rate, Network Delay and Power Consumption. The following subsections show the performance analysis of proposed model with Medium Access Control- Low Energy Adaptive Clustering Hierarchy (MAC-LEACH) [18] and conventional method when sending data with full data rate and using GPSR routing protocol for the three given scenarios. The result is represented in graphically forms.

\subsubsection{Packet Loss Rate}

From figure (4.7) it can be seen that the simulation result of the proposed model is achieves better performance than MACLEACH and the conventional method in term of packet loss rate parameter, for the three scenarios.

In addition, in figure (4.8) the proposed model has proven more efficient compared to MAC-EACH and the conventional method in $n$ case of packet loss rate as a function of time for the three scenarios.

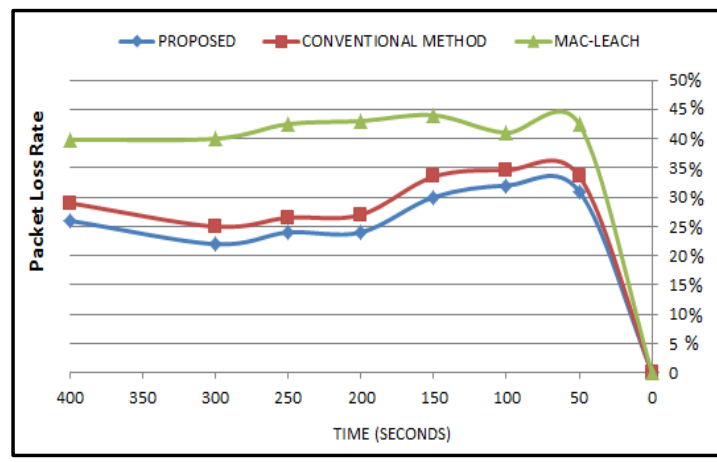

Figure(4.7): Average Packet Loss for Different Network Scenarios Using the Proposed Approach, the Conventional Method, and MAC-LEACH

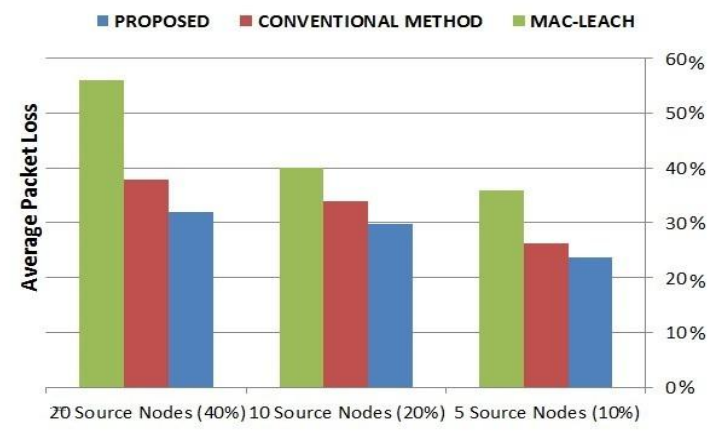

Figure (a)

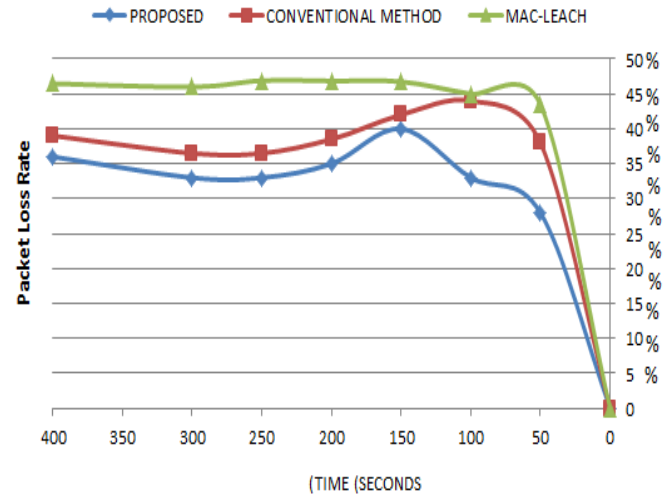

Figure (b)

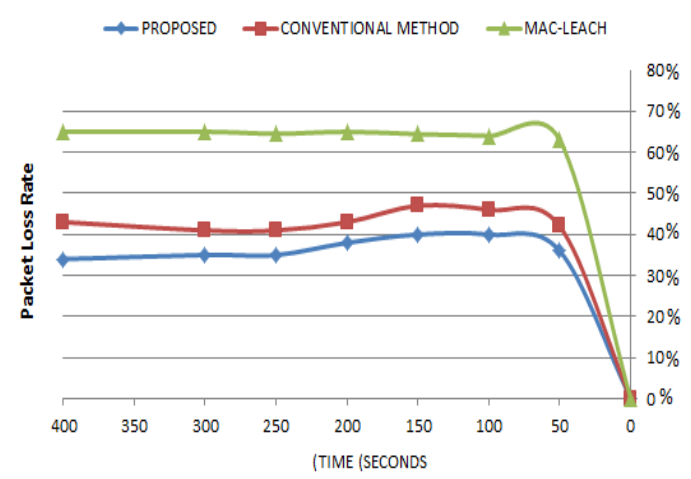

Figure (c)

Figure(4.8) : The Timeline of the Packet Loss Rate for Different Network Scenarios: (a) 10\% Source Nodes, (b) $20 \%$ Source Nodes, and (c) $40 \%$ Source Nodes

= PROPOSED = CONVENTIONAL METHOD M MAC-LEACH

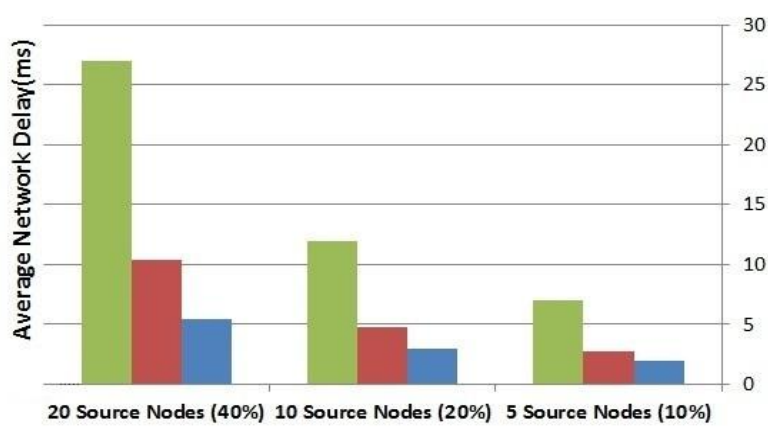

Figure(4.9) : Average Network Delay for Different Network Scenarios using the Proposed Approach, the Conventional Method, and MAC-LEACH 


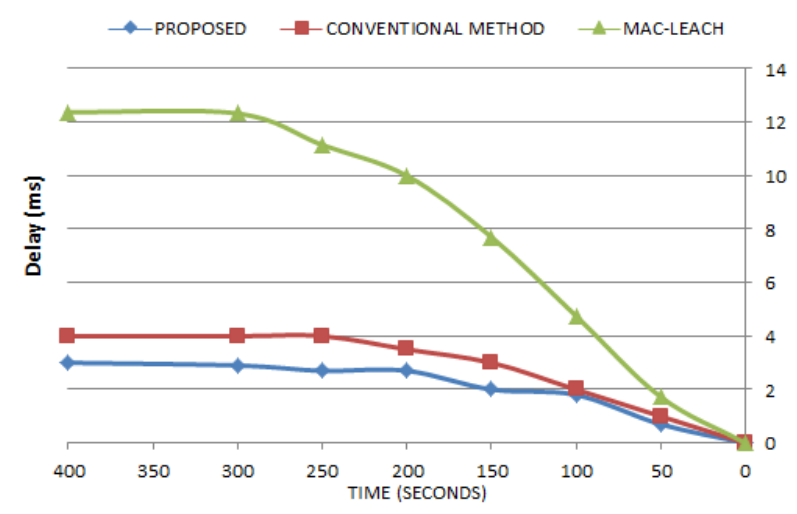

Figure (a)

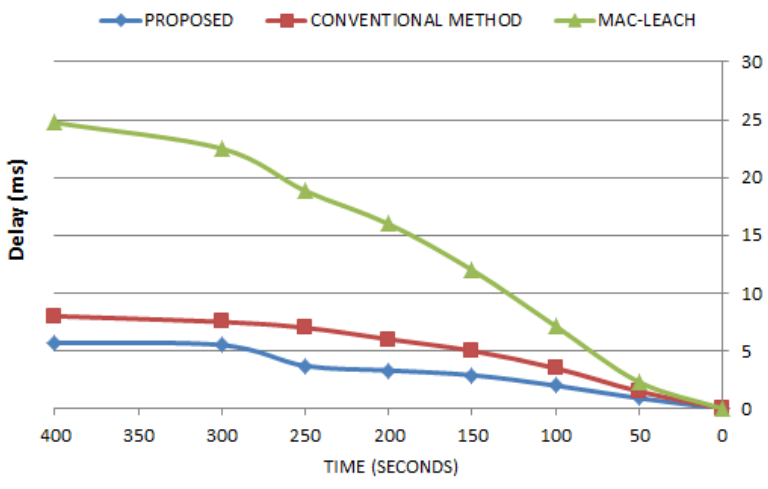

Figure (b)

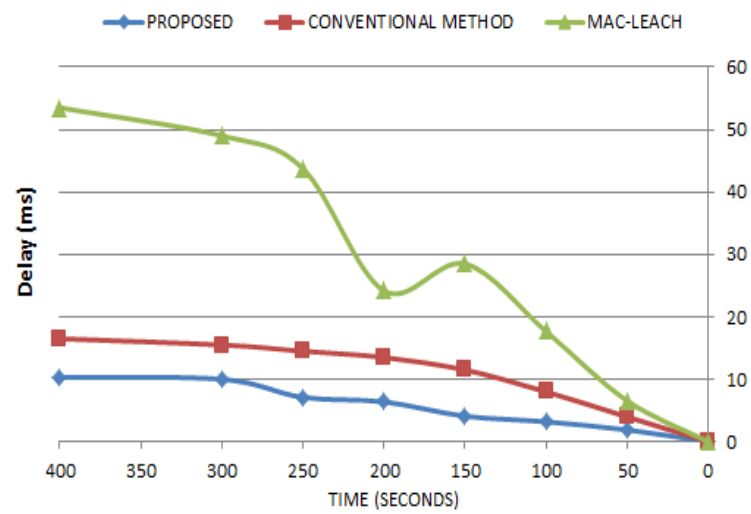

Figure (c)

Figure(4.10): The Timeline of the Delay for Different Network Scenarios: (a) $10 \%$ Source Nodes, (b) $20 \%$ Source Nodes, and (c) $40 \%$ Source Nodes

\subsubsection{Network Delay}

The average network delay parameter is depicted in the figure (4.9), also the figure (4.10) shows the delay rate as a function of time for the three scenarios. The simulation result shows that the proposed model is introduced lower delay compared to the other MAC-LEACH and the conventional method.

\subsubsection{Power Consumption}

Figure (4.11) shows the average power consumed for different network scenarios using the three schemes. As shown, the proposed approach achieves lower power consumption rate compared to MAC-LEACH and the conventional method. Figure (4.12) shows the power consumption rate as a function of time for the three scenarios. Moreover, the proposed approach has proven its efficiency compared to MAC-LEACH and the conventional method.

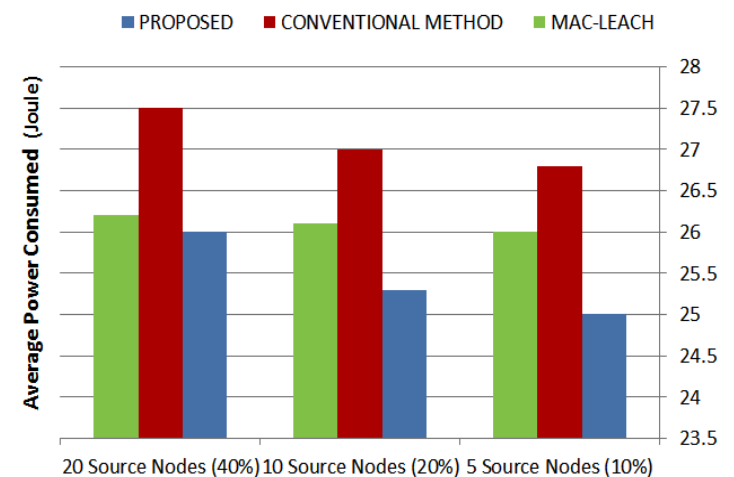

Figure(4.11): Average Power Consumed for Different Network Scenarios Using the Proposed Approach, the Conventional Method, and MAC-LEACH

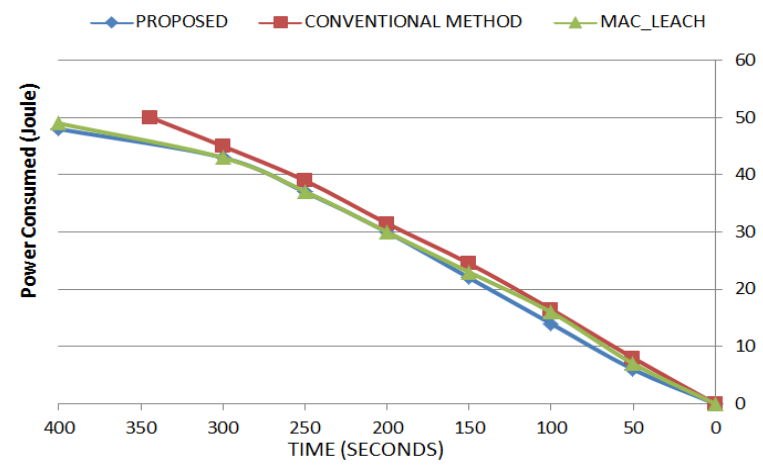

Figure (a)

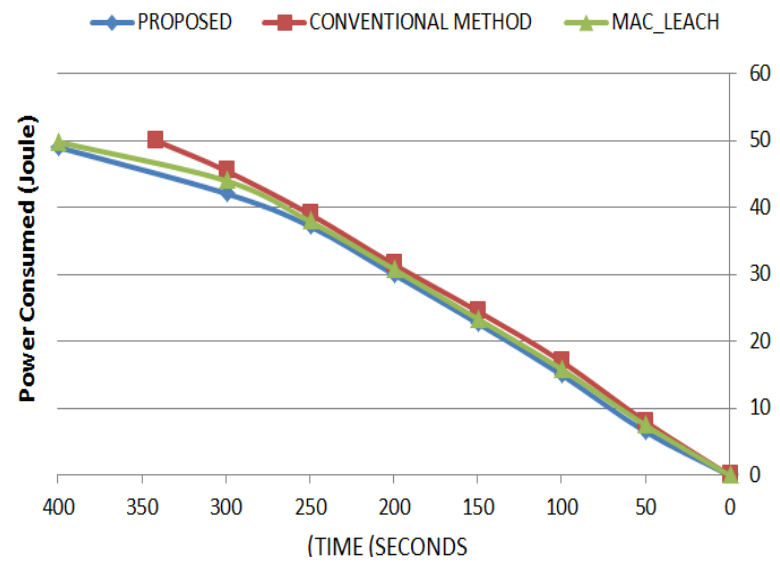

Figure (b)

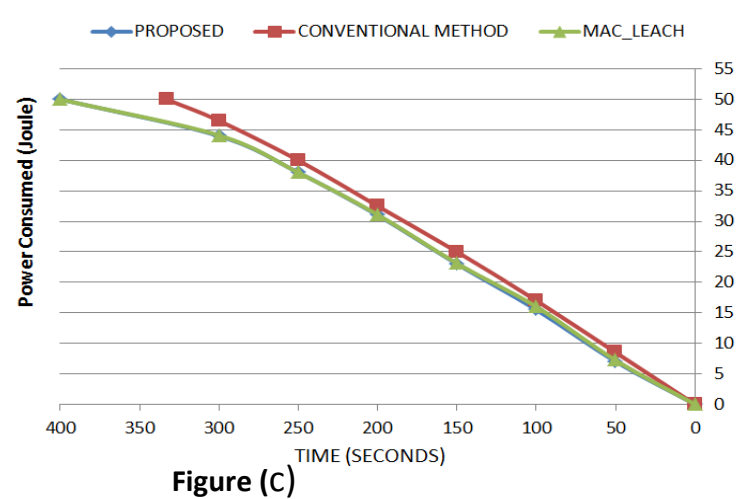

Figure(4.12) : The Timeline of the Power Consumed for Different Network Scenarios: (a) $10 \%$ Source Nodes, (b) $20 \%$ Source Nodes, and (c) $40 \%$ Source Nodes 


\section{CONCLUSION AND FURTHER STUDY}

In this paper, an efficient control system model was proposed to improve the QoS requirements in wireless sensor networks, which is reflected positively on IoTs. The proposed approach applies data rate adaptation and determines the appropriate routing protocol to work in the network according to the network conditions in order to improve the QoS parameters and maximize the resource utilization. Adapting the data rate and determining the appropriate routing protocol are carried out by control system model, which consists of fuzzy logic and binary controllers based on the instantaneous conditions of the network. In order to evaluate the proposed control system model performance, the data transmission process was simulated using real data (real images) with taking into account different network scenarios. Castalia 3.2 and XEEMU-1.0 simulators with CJPEG software were used to simulate all the operations starting with image capturing until displaying the received images at the base station.

The simulation results showed the efficiency of the proposed control system model in terms of power consumption, delay, and loss rate compared to the other schemes as MAC-LEACH, in addition to the conventional method when sending data with full data rate and using GPSR routing protocol. The future is to implement the proposed system using Non-Static Wireless Sensor Networks and to study the security factor in network when using SWARM protocol.

\section{REFERENCES}

[1] O. Vermesan, P. Friess, P. Guillemin, H. Sundmaeker, et al., (2013), "Inter- net of Things Strategic Research and Innovation Agenda", River Publishers, ISBN 978-8792982-73-5.

[2] Salem Belhaj, Sofian Hamad,(2018), "Routing protocols from wireless sensor networks to the internet of things: An overview", International Journal of Advanced and Applied Sciences, Pages: 47-63.

[3] Varun Sharma, and Somesh Sharma, (2017)," Low Energy Consumption based Patient Health Monitoring by LEACH Protocol" International Conference on Inventive Systems and Control (ICISC).

[4] Youngbok Cho, Minkang Kim, and Sunghee Woo, (2018), " Energy Efficient IoT based on Wireless Sensor Networks for Healthcare " International Conference on Advanced Communications Technology(ICACT).

[5] Yan Sun, Junpeng Guo, and Yuhui Yao,( July 2017), "Speed Up-Greedy Perimeter StatelessRouting Protocol for Wireless Sensor Networks (SU-GPSR)", IEEE.

[6] Anupkumar M Bongale, et al ,(2017)," EiP-LEACH: Energy influenced Probability based LEACH Protocol for Wireless
Sensor Network", International Conference on Emerging Trends \& Innovation in ICT (ICEI).

[7] HONGYAN XIN, AND XUXUN LIU,( September 2017)," Energy-Balanced Transmission With Accurate Distances for Strip-Based Wireless Sensor Networks", IEEE.

[8] Hui Chen, Feng Wang, and Mu Zhang,( 2017 ), "An Improved Inter-Cluster Multi-hop LEACH Protocol Oriented Reliable Transmission in WSNs" IEEE International Conference on Computer and Communications

[9] Hasna Dhehibi, et al,( 2017), " An Enhancement of MODLEACH protocol for Wireless Sensor Network ", International Multi-Conference on Systems, Signals \& Devices (SSD)

[10] Sabin Bhandari, et al ,( 2017 )," Cloud-Assisted Device Clustering for Lifetime Prolongation in Wireless IoT Networks" , IEEE 30th Canadian Conference on Electrical and Computer Engineering (CCECE).

[11] Mengkun , Huan , et al,( July 2017)," An Improved GPSR Protocol Based on Stratification of Traffic Density", International Conference on Information and Automation (ICIA) Macau SAR, China.

[12] Moad Mowafi, et al ,(2018),"Fuzzy Logic Based CrossLayer Design for Wireless Multimedia Sensor Networks", International Journal of Computational Intelligence Systems.

[13] Padmalaya Nayak, and D. Anurag,(2015) "A Fuzzy Logic based Clustering Algorithm for WSN to extend the Network Lifetime ", DOI 10.1109/JSEN.2472970, IEEE Sensors Journal.

[14] Prof. Elmer Dadios (Ed.), (2012), A Mamdani Type Fuzzy Logic Controller, Fuzzy Logic - Controls, Concepts, Theories. AndApplications, ISBN: 978-953-51-0396-7. http://web.univ-pau.fr/ cpham/WSN-MODEL/wvsn.html

[15] The Castalia simulator http://castalia.research.nicta.com.au/index.php/en

[16] N.K.R. Johari,( july 2012), "Power Profiling and Analysis of MI-Benchmarks Using Xscale Power Simulator (XEEMU)," Global Journals Inc.(USA), vol.12, no. 7.

[17] OMNeT++ network simulation framework. http://www.omnetpp.org/

[18] Thi Nguyen, et al,(2019),"Enhancing Energy Efficiency of WSNs through a novel Fuzzy Logic based on LEACH Protocol",International Symposium on Communications and Information Technologies (ISCIT) 